\title{
Parameterizing the Leaching Surface by Combining Curve-Fitting for Solute Breakthrough and for Spatial Solute Distribution
}

\author{
E. Bloem • M. de Gee • G. H. de Rooij
}

Received: 10 August 2010 / Accepted: 13 December 2011 / Published online: 22 January 2012

(C) The Author(s) 2012. This article is published with open access at Springerlink.com

\begin{abstract}
Multi-compartment samplers (MCSs) measure unsaturated solute transport in space and time at a given depth. Sorting the breakthrough curves (BTCs) for individual compartments in descending order of total solute amount and plotting in $3 \mathrm{D}$ produces the leaching surface. The leaching surface is a useful tool to organize, present, and analyze MCS data. We present a novel method to quantitatively characterize leaching surfaces. We fitted a mean pore-water velocity and a dispersion coefficient to each BTC, and then approximated their values by functions of the rank order of the BTCs. By combining the parameters of these functions with those of the Beta distribution fitted to the spatial distribution of solutes, we described an entire leaching surface by four to eight parameters. This direct characterization method allows trends to be subtracted from the observations, and incorporates the effects of local heterogeneity. The parametric fit creates the possibility to quantify concisely the leaching behavior of a soil in a given climate under given land use, and eases the quantitative comparison of spatio-temporal leaching behavior in different soils and climates.
\end{abstract}

E. Bloem

Soil Physics, Ecohydrology and Groundwater Management,

Environmental Sciences Group, Wageningen University, P.O. Box 47,

6700 AA Wageningen,

The Netherlands

E. Bloem ( $\varangle)$

Soil and Environment Division, Bioforsk, Norwegian

Institute for Agricultural and Environmental Research,

Frederik A. Dahls vei 20,

1432 Ås, Norway

e-mail: Esther.Bloem@Bioforsk.no

M. de Gee

Biometris, Wageningen University, P.O. Box 100,

6700 AC Wageningen, The Netherlands

G. H. de Rooij

Soil Physics Department, Helmholtz Centre for Environmental Research — UFZ,

Theodor-Lieser-Strasse 4, 06120 Halle (Saale), Germany 
Keywords Solute transport - Unsaturated zone - Groundwater contamination • Breakthrough curve $\cdot$ Spatio-temporal distribution · Leaching surface

Beta distribution · Curve-fitting

$\begin{array}{ll}\text { Abbreviations } \\ \text { BTC } & \text { Breakthrough curve } \\ \text { CDE } & \text { Convection-dispersion equation } \\ \text { CV } & \text { Coefficient of variation } \\ \text { EC } & \text { Electrical conductivity } \\ \text { MCS } & \text { Multi-compartment sampler } \\ \text { RMSE } & \text { Root mean square error } \\ \text { SSDC } & \text { Spatial solute distribution curve } \\ \text { STDEV } & \text { Standard deviation }\end{array}$

\section{List of symbols}

$a_{\mathrm{D}} \quad$ Fitting parameter

$a_{\mathrm{v}} \quad$ Fitting parameter

A Sample collection area $\left(\mathrm{L}^{2}\right)$

$b_{\mathrm{D}} \quad$ Fitting parameter

$b_{\mathrm{v}} \quad$ Fitting parameter

$B$ Beta function

$c_{\mathrm{D}} \quad$ Fitting parameter

$c_{\mathrm{V}} \quad$ Fitting parameter

C Solute flux concentration $\left(\mathrm{ML}^{-3}\right)$

$C^{*} \quad$ Scaled solute flux concentration $\left(\mathrm{T}^{-1}\right)$

$C_{0} \quad$ Area under breakthrough curve $\mathrm{BTC}_{C}\left(\mathrm{ML}^{-3} \mathrm{~T}\right)$

$C_{\mathrm{f}} \quad$ Dimensionless flux-averaged concentration (-)

$D \quad$ Dispersion coefficient $\left(\mathrm{L}^{2} \mathrm{~T}^{-1}\right)$

$F \quad$ Solute flux density $\left(\mathrm{ML}^{-2} \mathrm{~T}^{-1}\right)$

$F_{\mathrm{O}}$ Observed solute flux density $\left(\mathrm{ML}^{-2} \mathrm{~T}^{-1}\right)$

$F_{\mathrm{P}} \quad$ Calculated solute flux density $\left(\mathrm{ML}^{-2} \mathrm{~T}^{-1}\right)$

$k \quad$ Sampling time index

$n \quad$ Number of cells $(-)$

$m \quad$ Total number of sampling rounds

$p \quad$ Probability of the Beta variate as a function of coordinate $x$

$q \quad$ Water flux density $\left(\mathrm{LT}^{-1}\right)$

$q \quad$ Water flux density matrix $\left(\mathrm{LT}^{-1}\right)$

$\frac{q}{R} \quad$ Retardation factor (-)

$s \quad$ Cumulative sampling area $\left(\mathrm{L}^{2}\right)$

$S \quad$ Leaching surface $\left(\mathrm{ML}^{-2} \mathrm{~T}^{-1}\right)$

$t \quad$ Time $(\mathrm{T})$

$t_{k} \quad$ Time (T) at which the $k$ th set of samples was retrieved

$v \quad$ Pore-water velocity $\left(\mathrm{LT}^{-1}\right)$

$V \quad$ Water volume $\left(\mathrm{L}^{3}\right)$

$x_{i} \quad$ Horizontal Cartesian coordinate $(i$ is counter $1, \ldots, n)$

$y_{i} \quad$ Horizontal Cartesian coordinate $(i$ is counter $1, \ldots, n)$

$z \quad$ Depth below soil surface (L) 

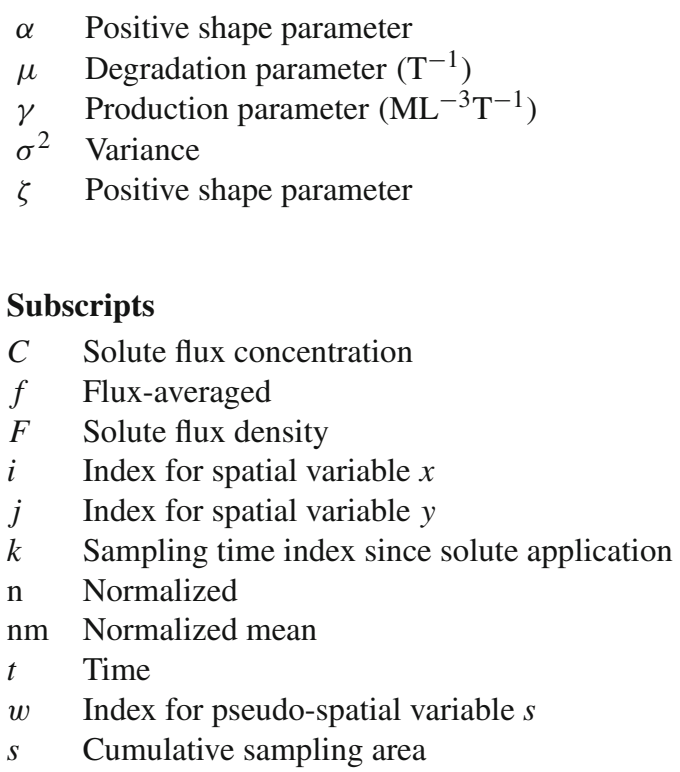

\section{Introduction}

Solute transport in soils is strongly affected by soil heterogeneity, fingered flow and macropore flow. The heterogeneity influences both water transport and solute transport, but not necessarily to the same extent. Dye tracers such as Brilliant Blue are often used for flow visualization (Flury et al. 1994; Flury and Wai 2003), and increasingly for quantitative analysis (Persson 2005). While dye tracers provide information about spatial spreading, the significant adsorption make these tracers less suited to determine the travel times of water (Kasteel et al. 2002). Another disadvantage of using dye is that it allows only a single experiment at a given location.

Travel times of water and solutes can be studied efficaciously using column studies where conservative tracers like chloride or bromide are used to measure the temporal response to the injected tracer. In a column experiment, a solute moves downward with the water through a single column. At the bottom, water and solute are collected in a single container covering the entire bottom of the column, and the measurements result in a breakthrough curve (BTC) (Jury and Roth 1990). These single column experiments lack spatial information and the BTC essentially describes one-dimensional solute transport. Field experiments with singlecell sample collectors also give a single (spatially averaged) BTC, and therefore only give information about solute transport in one direction.

This limitation prompted the ever-increasing deployment of multi-compartment samplers (MCS) that measure solute leaching as a function of space and time (Wildenschild et al. 1994; Quisenberry et al. 1994; Poletika and Jury 1994; Buchter et al. 1995; Stagnitti et al. 1998; de Rooij and Stagnitti 2000; Strock et al. 2001; Bloem et al. 2010) to assess the fate of salts, nutrients, and pollutants in natural, heterogeneous soils. The MCS we used in our experiment is designed by Bloem et al. (2010), which can be installed in situ in a tunnel dug below an undisturbed soil volume. The top of the MCS consists of a horizontal flat sampling plate of porous material. The plate is divided into 100 compartments, each with a size of 
$31.5 \times 31.5 \mathrm{~mm}$. Drop counters below the top plate record the number of drops passing through each compartment every $5 \mathrm{~min}$, giving the water flux per compartment. The percolate passing through each compartment is retained separately for each compartment. This percolate can be repeatedly extracted while leaving the instrument buried in situ, facilitating prolonged operation times. To minimize the disturbance of the flow field, the MCS is capable of modifying the applied suction to correspond to the ambient pressure head, as measured by tensiometers installed at the same depth as the instrument. By carrying out a tracer experiment and determining the volume and composition of each extracted sample, the BTCs for all individual compartments can be determined. Therefore, the output of a multi-compartments sampler consists of a family of BTCs.

An individual BTC generally has been interpreted through moment analysis (Kreft and Zuber 1978; Vanderborght and Vereecken 2001; Brooks and Wise 2005) or by means of a solute transport model (model analysis) (Parker and van Genuchten 1984; Jury and Sposito 1985; Kool et al. 1987; Yamaguchi et al. 1989). Moment analysis usually considers the zeroth moment of the distribution of solute with time (recovered tracer mass), the first moment (mean travel time), and the second central moment (variance of the travel time). In model analysis, mathematical equations are used to describe solute transport. The parameters in these equations are matched to the measured response (curve-fitting). Thus, the transport properties of the system are represented by the model parameters (Toride et al. 1999). Fahim and Wakao (1982) and Haas et al. (1997) concluded that curve-fitting in a model analysis is more accurate than moment analysis. Furthermore, model analysis is often preferred, because the model parameters usually have a clear physical interpretation. This, however, may also be a pitfall: the inability of mathematical models to accurately describe the nature of complex systems can be a disadvantage in the model analysis approach (Brooks and Wise 2005).

The most common mathematical model for solute transport in soils is the convection-dispersion equation (CDE). The CDE characterizes solute transport by the velocity and dispersion coefficient. In soil physics the use of the CDE is accepted, even though it is acknowledged that soils have complex pore geometries and heterogeneous structures which are not fully known in detail (Beven et al. 1993). Therefore Beven et al. (1993) argue that the CDE may be applicable in a functional sense in which the mean transport velocity reflects the mass flux of water averaged over some unit area in the system, and the 'effective' dispersion coefficient accounts for the complexities of the flow pathways and heterogeneity in local fluid velocities in the direction of the flow. Modifications of the CDE have been made to improve this model. In saturated soils, the dispersion is scale dependent, necessitating the use of a scale-dependent coefficient (Mishra and Parker 1989; Gelhar et al. 1992). In unsaturated soils preferential flow exerts a large influence. This can be accounted for by using the mobile-immobile CDE model for example (Parker and van Genuchten 1984; Beven et al. 1993), or by models that include a macropore domain. Many studies have used model analysis for fitting the transport parameters to tracer data, (e.g., Parker and van Genuchten 1984; Jury and Sposito 1985; Kool et al. 1987; Yamaguchi et al. 1989), including the identifiability of the parameter values (e.g., Mishra and Parker 1989). A single BTC can only offer a limited amount of information and certain processes may be taking place outside of its unitary scope. The parameters of the mobile-immobile CDE, for example, may interact in their effect on the predicted outputs, resulting in considerable uncertainty associated with the fitted values (Beven et al. 1993).

Most experiments had a low spatial resolution and used only a single BTC. Therefore, they give poor representations of the heterogeneous transport processes. Tracer experiments with conservative tracers like chloride or bromide, in which multi-compartment samplers are used to measure the response to an injected tracer, are more adequate to characterize solute transport. A new approach to analyze this data has been developed (de Rooij and Stagnitti 
2002a,b, 2004), presenting the leaching surface as a tool to analyze the spatially and temporally non-uniform passage of solutes across a monitoring plane. The leaching surface is a curved surface constructed from a population of local-scale BTCs in such a way that the information on the spatial variation in the BTCs is preserved.

Until now, leaching surfaces were merely used to represent data from multi-compartment samplers. In this article, we develop a method to quantitatively characterize leaching surfaces. The main objective is to parameterize the leaching surface with a limited number of parameters that can be meaningfully interpreted in terms of the soil's solute transport properties. This is achieved by a separate parameterization of the spatial and temporal aspects of solute leaching. For the temporal aspect, we determine the parameters of the individual BTCs by model analysis. We then detect and parameterize any relationships between the transport velocities and dispersion coefficients of the individual BTCs. The spatial aspect is parameterized using the Beta distribution as outlined by Stagnitti et al. (1999) and de Rooij and Stagnitti (2000, 2004).

The best-fit parameters thus provide a quantitative and objective representation of the leaching surface. This allows a quantitative description of leaching surfaces, providing a means to objectively compare leaching characteristics of different soils, and even of the same soil in different seasons. To test the method, we performed a tracer experiment in the field with a multi-compartment sampler. We constructed a leaching surface from the data and determined its parameters.

\section{Materials and Methods}

\subsection{Leaching Surfaces: Spatial and Temporal Aspects of Solute Leaching}

In a hypothetical experiment, a multi-compartment sampler (MCS) with sample collection area $A\left(\mathrm{~L}^{2}\right)$ has its sampler cells arranged in a rectangular grid of $n \times n$ cells with positions $\left(x_{i}, y_{j}\right)$, where $x_{i}$ and $y_{j}(\mathrm{~L})$ are horizontal cartesian coordinates of the centers of $n^{2}$ individual cells identified by the counters $i, j \in\{1, \ldots, n\}$. Generalization to other geometries or a population of unconnected single-cell samplers is trivial. The sampler is buried in the soil below an undisturbed soil volume. For an experiment at which a solute is applied uniformly as a pulse (Dirac function) to the soil surface of an area much larger than $A$ centered above the sampler, the outflow into the cells is collected at several points $t_{k}(\mathrm{~T})$ in time, with subscript $k$ indicating the $k$ th sampling time since the solute application. Water volume $V\left(\mathrm{~L}^{3}\right)$ and solute flux concentration $C\left(\mathrm{ML}^{-3}\right)$ of the samples are measured.

For the solute, there are two ways in which to proceed. The first option is to use the measurements of concentration. These concentrations give a solute flux concentration breakthrough curve $\mathrm{BTC}_{C}\left(x_{i}, y_{i}, t\right)$ for compartment $(i, j)$. The second option is to use solute flux density. Multiplying the measurements of solute flux concentration $C$ and water volume $V$ results in a derived leached mass measurement. By dividing this mass by the compartment area and by the sampling time interval $t_{k}-t_{k-1}$ we obtain the solute flux density $F\left(\mathrm{ML}^{-2} \mathrm{~T}^{-1}\right)$

$$
F_{i, j, k}=\frac{C_{i, j, k} V_{i, j, k}}{\Delta x_{i} \Delta y_{j}\left(t_{k}-t_{k-1}\right)}
$$

We can then obtain the solute flux density breakthrough curve $\operatorname{BTC}_{F}\left(x_{i}, y_{i}, t\right)$ by plotting the solute flux density $F$ as a function of time. Each of these approaches has its merits and 
drawbacks. To describe solute transfer through the soil, $\mathrm{BTC}_{F}$ is most valuable. When the concentration is of interest (for instance for substances with non-linear sorption, or for toxic compounds), $\mathrm{BTC}_{C}$ is preferable.

Both functions contain the full spatial information gathered by the multi-compartment device. Since they are functions of three independent variables, it is difficult to analyze them. To facilitate their visualization, the sampling compartments are ranked in decreasing order of their total collected solute over the entire leaching period. In this way, the two spatial variables $x$ and $y$ are collapsed into one single pseudo-spatial variable $s\left(\mathrm{~L}^{2}\right)$, such that each compartment corresponds to a length of $s$ that is proportional to its sampling area. Of course, this ranked variable $s$ can be related back uniquely to the original spatial variables $x$ and $y$ by two indexing functions index_ $x$ and index_y, such that the compartment with ranked coordinate $s$ corresponds to the physical compartment with coordinates $x$ index $\_x(s)$ and $y$ index_y $y(s)$. Next, the corresponding breakthrough functions $\mathrm{BTC}_{F}$ or $\mathrm{BTC}_{C}$ are plotted against $s$. This gives a plot of adjacent breakthrough curves that together form a curved surface. When this surface is constructed from $\mathrm{BTC}_{F} \mathrm{~s}$, it is termed the leaching surface, which was introduced by de Rooij and Stagnitti (2002a)

$$
S(s, t)=F\left(x \text { index } \_x(s), y \text { index_y }(s), t\right)
$$

The leaching surface $S(s, t)\left(\mathrm{ML}^{-2} \mathrm{~T}^{-1}\right)$ thus has a horizontal time-axis and a second horizontal axis $\left(s\left(\mathrm{~L}^{2}\right)\right)$ that represents the cumulative area of the compartments into which the control plane is divided. In the ordering, the detailed spatial information is lost, but the variation remains. The leaching surface can be scaled to make the area underneath it equal to one. Suitable cross-sections of the leaching surface parallel to the time-axis or the spatial axis, or integrations along intervals of the space and the time coordinate, can yield a wealth of information about the distribution in space and time of solute movement. Indeed, at any fixed point $s$ (associated with a location $\left(x_{i}, y_{j}\right)$, the cross-section of the $S$ parallel to the $t$-axis returns the solute flux density breakthrough curve at $\left(x_{i}, y_{j}\right)$. Integration over $t$ gives the spatial solute distribution curve

$$
\operatorname{SSDC}(s)=\int_{0}^{\infty} S(s, t) \mathrm{d} t
$$

By definition, this is a non-negative monotonically decreasing function. It reflects the spatial redistribution of a uniformly applied solute (Stagnitti et al. 1999; de Rooij and Stagnitti 2004).

\subsection{Parameterizing the Temporal Aspect of Solute Leaching}

We seek a practical method of determining solute spreading in each compartment. To do so we simplify the flow to steady-state by replacing the time coordinate by a transformed time that represents the cumulative amount of drainage (L). For clarity of presentation we retain the notation $t$ in all equations used in this article and note that the time coordinate used in the derivations is truly a transformed time coordinate that makes a unit of time directly proportional to a unit of drainage. Each $\mathrm{BTC}_{C}$ is scaled to 1 by dividing the concentrations by $C_{0}\left(\mathrm{ML}^{-3} \mathrm{~T}\right)$ (the area under each $\left.\mathrm{BTC}_{C}\right)$. We model the scaled $\mathrm{BTC}_{C}\left(\mathrm{~T}^{-1}\right)$ for each cell as the solution of a conventional one-dimensional equilibrium CDE, without modifications (Toride et al. 1999). The transformed time coordinate ensures that the value of $C_{0}$ is proportional to the solute mass passed. Thus, the solute transport is characterized by the pore-water velocity $v\left(\mathrm{LT}^{-1}\right)$, dispersion coefficient 
$D\left(\mathrm{~L}^{2} \mathrm{~T}^{-1}\right)$, retardation factor $R(-)$, and degradation or production parameters $\mu\left(\mathrm{T}^{-1}\right)$, and $\gamma\left(\mathrm{ML}^{-3} \mathrm{~T}^{-1}\right)$

$$
R \frac{\partial C}{\partial t}=D \frac{\partial^{2} C}{\partial z^{2}}-v \frac{\partial C}{\partial z}-\mu C+\gamma(z)
$$

with $z$ denoting depth below the soil surface (L), and $C$ the solute flux concentration $\left(\mathrm{ML}^{-3}\right)$.

In our experiment we used a conservative tracer, thus $R=1, \mu=0$, and $\gamma=0$. For input we used the Dirac delta pulse. Furthermore, we measured flux-averaged concentrations. To allow an analytical solution we assumed steady-state flow. We used a boundary value problem condition consistent with our flux concentration observations. The specific solution for this problem is given by Toride et al. (1999)

$$
C^{*}(t)=\left(\frac{L^{2}}{4 \pi D t^{3}}\right)^{\frac{1}{2}} \exp \left(-\frac{(L-v t)^{2}}{4 D t}\right)
$$

with $C^{*}$ is the scaled flux concentration $\left(\mathrm{T}^{-1}\right)$ and $L$ denoting the depth at the sampling area (L).

As Toride et al. (1999) stated, this solution is sometimes referred to as the travel time probability density function (pdf) for the equilibrium CDE (Jury and Roth 1990). The parameters $v$ and $D$ were fitted using CXTFIT (Toride et al. 1999), a computer program for estimating solute transport parameters from observed concentrations using the convection-dispersion equation (CDE) as the transport model. The CXTFIT code uses a nonlinear least-squares parameter optimization method for estimating the solute transport parameters. For our hypothetical experiment, we obtain $n^{2} \mathrm{BTC}_{C} \mathrm{~s}$, each with its own $v$ and $D$. To have a workable number of parameters we adopted simple functional relationships between $v$ and $D$ on one hand and $s$ on the other

$$
\begin{gathered}
v(s)=a_{\mathrm{v}} s^{b_{\mathrm{v}}}+c_{\mathrm{v}} \\
D(s)=a_{\mathrm{D}} s^{b_{\mathrm{D}}}+c_{\mathrm{D}}
\end{gathered}
$$

where $a_{\mathrm{v}}, a_{\mathrm{D}}, b_{\mathrm{v}}, b_{\mathrm{D}}, c_{\mathrm{v}}$, and $c_{\mathrm{D}}$ are fitting parameters. The chosen functions allow both linear and power law fits, but can of course be replaced by any desired relationship. Also, constraints linking some of the parameters can be added to reduce the number of fitting parameters. As a starting point we defined a reference fit by setting $c_{\mathrm{V}}$ and $c_{\mathrm{D}}$ to the mean fitted pore-water velocity and dispersion coefficient, respectively, with the remaining fitting parameters set to zero.

We chose to fit scenario dependent dispersion coefficients rather than more stable and soildependent dispersivities for clarity: the aim was to characterize the leaching surface itself, not the soil that generated it. More fundamentally, the soil volume for which a dispersivity is determined, and which presumably is characterized by it, can only be accurately known for column or lysimeter experiments with single outlets. Different initial and boundary conditions do not affect this soil volume. For MCS experiments, on the other hand, the dispersivity determined for an individual sampling compartment characterizes the stream tube exiting through that compartment, and a change in the initial and boundary conditions is likely to result in a different flow pattern with reconfigured stream tubes. One may argue that the population of dispersivities still characterizes the soil volume contained by the entire population of stream tubes, and that this volume is likely to change much less with changing initial and boundary conditions. Still, it is unlikely and certainly not a prerequisite that the distribution of dispersivities is independent of initial and boundary conditions. Thus, the parameters describing 
the distribution of dispersivities would still be scenario dependent. To avoid the suggestion that we characterize not only the solute plume but also the soil we therefore refrained from calculating the individual dispersivities.

\subsection{Parameterizing the Spatial Aspect of Solute Leaching}

The various single-cell BTCs produced by a MCS differ because of soil spatial variation and possibly preferential flow. It is desirable to characterize the effect of soil heterogeneity on solute leaching directly (i.e., not through numerical simulations in which the soil hydraulic properties vary between nodes). When MCS data are available, a simple yet descriptive way of doing so is through the SSDC, which is obtained by ranking the sampling compartments in decreasing order of amount of total captured solute and plotting the fraction of captured solute as a function of the cumulative area of the ordered compartments (Quisenberry et al. 1994; Stagnitti et al. 1999). Thus, the SSDC only contains information about the spatial redistribution of solutes. If lateral dispersion is of minor importance, de Rooij and Stagnitti (2000) showed that the SSDC describes the geometry of the flow paths in terms of flow constriction and divergence. The SSDC can be parameterized by fitting the Beta distribution (Stagnitti et al. 1999)

$$
p(x, \alpha, \zeta)=B(\alpha, \zeta) x^{\alpha-1}(1-x)^{\zeta-1}, \quad 0 \leq x \leq 1
$$

where $p$ is the probability of the Beta variate as a function of coordinate $x$,

$$
B(\alpha, \zeta)=\frac{\Gamma(\alpha+\zeta)}{\Gamma(\alpha) \Gamma(\zeta)}
$$

is the Beta function, and $\alpha$ and $\zeta$ are positive shape parameters (Nadarajah and Gupta 2004). The mean, variance and coefficient of variation CV of the Beta distribution are (Gupta and Nadarajah 2004)

$$
\begin{aligned}
\operatorname{mean}(\alpha, \zeta) & =\frac{\alpha}{\alpha+\zeta} \\
\sigma^{2}(\alpha, \zeta) & =\frac{\alpha \zeta}{(\alpha+\zeta)^{2}(\alpha+\zeta+1)} \\
\mathrm{CV}(\alpha, \zeta) & =\sqrt{\frac{\zeta}{\alpha(\alpha+\zeta+1)}}
\end{aligned}
$$

Note that the uniform distribution arises by setting $\alpha=\zeta=1$, with a coefficient of variation $3^{-1 / 2}$.

We needed to transform the fitted $\mathrm{BTC}_{C}$ for each compartment $(i, j)$ to a $\mathrm{BTC}_{F}$ in order to be able to produce a leaching surface based on solute flux density. We approximated the flux concentration during the $k$ th sampling interval by

$$
C_{i, j, k} \approx C_{0, i, j} C_{i, j}^{*}\left(t_{k}\right)
$$

i.e., based on the fitted scaled concentration at the end of the sampling interval. With Eq. 1, this gives

$$
F_{\mathrm{i}, \mathrm{j}, \mathrm{k}} \approx \frac{C_{0, i, j} C_{i, j}^{*}\left(t_{k}\right) V_{i, j, k}}{\Delta x_{i} \Delta y_{j}\left(t_{k}-t_{k-1}\right)}
$$

The group $V_{i, j, k}\left[\Delta x_{i} \Delta y_{j}\left(t_{k}-t_{k-1}\right)\right]^{-1}$ is the water flux density for compartment $(i, j)$ during sampling interval $k$. We recall that we ranked the $n^{2}$ compartments in order of total 
captured solute, so that each compartment $(i, j)$ has a unique rank number, which we denote $\mathrm{w}: \mathrm{w} \epsilon\left\{1, \ldots, n^{2}\right\}$. If we denote the total number of sampling rounds $m$, we have $k \epsilon\{1, \ldots, m\}$. With these, we can create a water flux density matrix $q$ with $n^{2}$ rows and $m$ columns in which each of the entries is given by the water flux density of the compartment corresponding to the row number (ranking along the $s$-axis) at the sampling round indicated by the column. By summing over the columns we obtain the vector $\underline{q}_{s}\left(\mathrm{LT}^{-1}\right)$

$$
q_{s, \mathrm{w}}=\sum_{k=1}^{m} q_{\mathrm{w}, k}
$$

By summing over the rows we obtain the vector $\underline{q}_{t}\left(\mathrm{LT}^{-1}\right)$

$$
q_{t, k}=\sum_{\mathrm{w}=1}^{n^{2}} q_{\mathrm{w}, k}
$$

We now approximate the solute flux density in the compartment with rank number $\mathrm{w}$ at sampling interval $k$ as

$$
F_{\mathrm{w}, k} \approx C_{0, \mathrm{w}} C_{\mathrm{w}, k}^{*} q_{s, \mathrm{w}} \frac{q_{t, k}}{\sum_{k=1}^{m} q_{t, k}}
$$

Here, $C_{0, \mathrm{w}} q_{s, \mathrm{w}}$ approximates the total amount of solute leached from the compartment with rank number w. It is a function of $\mathrm{w}$, and this function can be parameterized by the Beta distribution in analogy with the SSDC.

The Beta distribution fitted on $C_{0, \mathrm{w}} q_{s, \mathrm{w}}$ gives the fraction of the total leached solute passing through a particular region associated with an interval $\mathrm{d} s$. This provides a scale factor to adjust the scaled breakthrough curve $\mathrm{BTC}_{F}$ with $v(s)$ and $D(s)$ for that location. In fitting the Beta distribution to approximate total amounts of leaching, we fitted the non-cumulative form of the distribution. We found this significantly improved the goodness of fit as compared to fitting the cumulative Beta distribution according to de Rooij and Stagnitti (2000).

\subsection{Leaching Experiment in the Field}

We used a variable-suction multi-compartment sampler of the type developed by Bloem (2008) and Bloem et al. (2010) with metal porous plates (metal sampler) (Fig. 1). The sampler consisted of $10 \times 10$ compartments $(n=10)$, each with a sampling area of $10.35 \mathrm{~cm}^{2}$. The sampler was installed in the field (Vredepeel, the Netherlands) at $31 \mathrm{~cm}$ depth as described by Bloem (2008) and Bloem et al. (2009). On the 14th of December 2005 we cut the grass in the $0.70 \times 0.70 \mathrm{~m}$ area above the sampler to $3 \mathrm{~cm}$ and applied $4.5 \mathrm{~mm}$ of a $1 \mathrm{M} \mathrm{CaCl}_{2} \cdot 2 \mathrm{H}_{2} \mathrm{O}$ solution. To eliminate the side effects of both converging and diverging streamlines of the chloride concentration, the tracer solution was applied on the entire $0.70 \times 0.70 \mathrm{~m}$ plot. To do this, we covered the application area with a $21 \times 21$ cell PVC grid, with a syringe holder in the center of each cell. We filled 441 medical $10 \mathrm{ml}$ syringes with $5 \mathrm{ml}$ tracer solution $(\mathrm{CV}$ $=0.7 \%$ ), and placed these in the syringe holders. We then emptied all syringes within $2 \mathrm{~min}$ to achieve a spatially uniform tracer pulse application. The soil surface directly above the sampling area of the sampler received $33.0 \mathrm{~g} \mathrm{Cl}^{-}$.

After each rainfall event (usually a cluster of small rain showers), the collected leachate was extracted from the sampling cells while leaving the sampler buried in situ. The collected volumes were determined and the solute concentrations were derived from the EC as measured with an EC meter (Cond 315i and TetraCon325 from WTW; individually calibrated). 

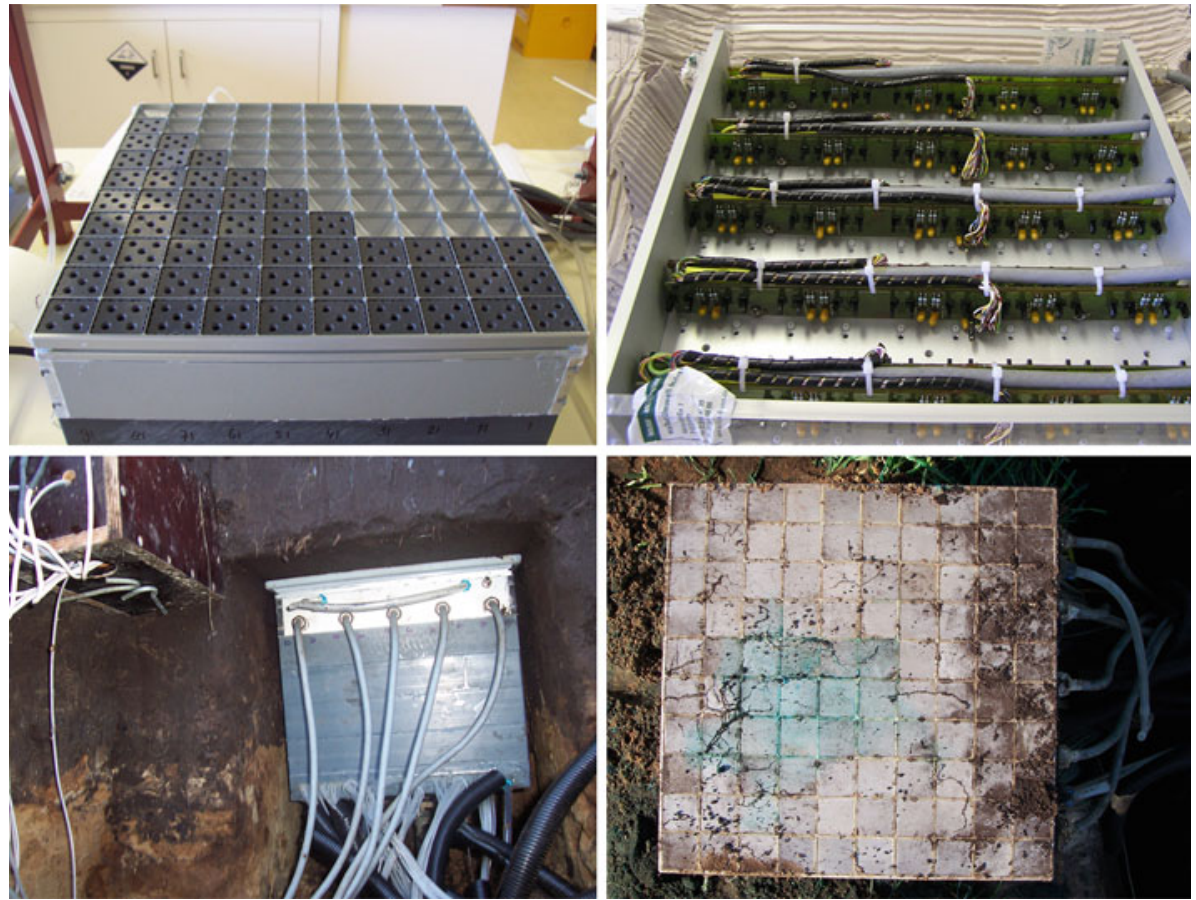

Fig. 1 Construction details and key features of the multi-compartment sampler (MCS). The instrument has high-spatial resolution with 100 sampling compartments and high temporal resultion of suction, rainfall measurements, and drop counts. To the instrument vacuum is applied which follows ambient suction. The cover of the instrument consists of porous metal plates

After 145 days (11 sampling rounds; $k \in 1, \ldots, 11$ ), nearly all tracer had passed the sampling depth. The mass collected during the final sampling round was less than $0.01 \%$ of the applied mass over the sampling area. In total the metal sampler captured $67 \%$ of the chloride it should have intercepted if the flow had been perfectly vertical.

\subsection{Data Analysis}

Rainfall during the experimental period was erratic. We therefore replaced the time coordinate $(\mathrm{T})$ by a cumulative drainage coordinate $(\mathrm{L})$, with the cumulative drainage at each sampling round calculated as the average cumulative drainage of all 100 sampling cells since the solute application. This coordinate transformation eliminates most of the irregularities arising from dry periods between rainfall events (van Ommen et al. 1989; Jury et al. 1991). We determined $\mathrm{BTC}_{C}$ and $\mathrm{BTC}_{F}$ for each compartment and constructed leaching surfaces from both sets of curves. For each leaching surface the $x$-axis consists of the cumulative drainage coordinate (L) and the $y$-axis consists of the cumulative area of the compartments $\left(\mathrm{L}^{2}\right)$. Each compartment covers a sampling area of $10.35 \mathrm{~cm}^{2}$, thus with 100 compartments the $y$-axis range from 0 to $1,035 \mathrm{~cm}^{2}$. Each leaching surface comprised 1,100 data points. The next step was to fit the $\mathrm{BTC}_{C}$ s of all compartments to Eq. 5. Before doing so we scaled every curve by the area underneath it ( $C_{0}$ [mass per volume times cumulative drainage; $\mathrm{ML}^{-3} \mathrm{~L}$; note the separate length dimension emerging from the coordinate transformation outlined above]), and then fitted $v$ and $D$ to the scaled curves. The flux concentration for a 
given cell at a given time can be approximated by multiplying the fitted concentration by $C_{0}$ of that cell.

Samples which did not contain enough water to analyze them separately were pooled during the analysis. This results in an average concentration from the pooled samples. Ten samples hardly ever contained enough water and therefore show the same concentrations in their curves, and their BTCs are entirely determined by the average concentration of the pooled samples. As those samples contained hardly any mass they were ranked lowest and grouped next to each other in the leaching surface. The breakthrough curves are biased by the averaging and are therefore not taken into account for the further construction of the leaching surface.

The resulting velocities from the fitting procedure (Eq. 5) are then parameterized by Eq. 6. In this case the velocity can be described by a single parameter, the average velocity. The same holds for the dispersion coefficient. The resulting dispersion coefficients from the fitting procedure (Eq. 5) are parameterized by Eq. 7, resulting in a single dispersion coefficient, the average dispersion coefficient. We constructed the leaching surface with the average velocity and the average dispersion coefficient for all $\mathrm{BTC}_{C} \mathrm{~s}$.

For comparison we also constructed the leaching surface based on flux concentration directly from the 100 fitted values of $v$ and $D$. Thus, the only difference with the observed leaching surface based on flux concentration is the fact that the individual BTCs were now fitted, isolating the effect of the fitting procedure to the CDE (Eq. 5). Conversely, we scaled the observed $\mathrm{BTC}_{C} \mathrm{~s}$ according to the fitted instead of the observed approximated SSDC to see how the Beta distribution affected $S(s, t)$.

For each BTC within the leaching surface we calculated the normalized root mean square error (RMSE)

$$
\mathrm{RMSE}_{\mathrm{n}}=\frac{\sqrt{\sum_{k=1}^{m}\left(F_{O}(i, k)-F_{\mathrm{P}}(i, k)\right)^{2}}}{\sum_{k=1}^{m} F_{\mathrm{O}}(i, k)} \times 100 \%
$$

with subscript $\mathrm{O}$ denoting observed solute flux densities, and subscript $\mathrm{P}$ indicated their calculated counterparts.

We also calculated the normalized mean RMSE between the observed and fitted leaching surface

$$
\mathrm{RMSE}_{\mathrm{nm}}=\frac{\sum_{\mathrm{w}=1}^{n^{2}} \sqrt{\sum_{k=1}^{m}\left(F_{\mathrm{O}}(\mathrm{w}, k)-F_{\mathrm{P}}(\mathrm{w}, k)\right)^{2}}}{\sum_{\mathrm{w}=1}^{n^{2}} \sum_{k=1}^{m} F_{\mathrm{O}}(\mathrm{w}, k)} \times 100 \%
$$

The same equation is used to calculate the error of the flux concentration $C$ (i.e., $F$ is replaced by $C$ in Eqs. 18 and 19).

\section{Results and Discussion}

Table 1 presents the day nr, cumulative infiltration (based on rainfall minus calculated evapotranspiration), and measured cumulative drainage in the sampler for the sampling period. For three selected sampling rounds (days 20,65, and 83) we present the collected water volume $\mathrm{V}\left(\mathrm{L}^{3}\right)$, its solute flux concentration $\mathrm{C}\left(\mathrm{M} \mathrm{L}^{-3}\right)$, and the mass measured in the individual compartments (Fig. 2). During the measured period the spatial variation of drainage is quite consistent. As can be seen in the figure, small-scale variations are abundant in the captured amounts of drainage. The captured volumes of adjacent cells can be very different, but clusters of high- and low-yielding cells are observed. Within the chloride flux concentration there 
Table 1 Percolation sampling dates, and rainfall, potential evapotranspiration, net infiltration, cumulative net infiltration, captured percolation, and cumulative captured percolation for each sample collection period

\begin{tabular}{llrrrrr}
\hline Day & $\begin{array}{l}\text { Rainfall } \\
(\mathrm{mm})\end{array}$ & $\begin{array}{l}\text { Potential evapo- } \\
\text { transpiration } \\
(\mathrm{mm})\end{array}$ & $\begin{array}{l}\text { Net infiltration } \\
(\mathrm{mm})\end{array}$ & $\begin{array}{l}\text { Cumulative net } \\
\text { infiltration } \\
(\mathrm{mm})\end{array}$ & $\begin{array}{l}\text { Drainage } \\
(\mathrm{mm})\end{array}$ & $\begin{array}{l}\text { Cumulative } \\
\text { drainage } \\
(\mathrm{mm})\end{array}$ \\
\hline 6 & 17.60 & 1.25 & 16.35 & 16.35 & 13.14 & 13.14 \\
20 & 12.40 & 3.00 & 9.40 & 25.75 & 13.46 & 26.60 \\
42 & 20.10 & 6.65 & 13.45 & 39.20 & 11.57 & 38.17 \\
58 & 15.40 & 5.20 & 10.20 & 49.40 & 8.22 & 46.39 \\
65 & 26.70 & 2.60 & 24.10 & 73.50 & 14.96 & 61.35 \\
70 & 30.40 & 1.55 & 28.85 & 102.35 & 30.22 & 91.57 \\
83 & 11.60 & 10.60 & 1.00 & 103.35 & 8.44 & 100.01 \\
86 & 24.40 & 0.95 & 23.45 & 126.80 & 11.61 & 111.62 \\
104 & 22.50 & 22.10 & 0.40 & 127.20 & 6.59 & 118.21 \\
112 & 23.80 & 11.70 & 12.10 & 139.30 & 10.05 & 128.26 \\
145 & 27.90 & 48.70 & -20.80 & 118.50 & 5.08 & 133.34 \\
\hline
\end{tabular}

is less spatial variation than in the drainage pattern, reflecting the effect of lateral spreading. The distribution of chloride mass resembled the drainage pattern.

Figure 2 also presents the total amount of water, maximum concentration, and total mass for the 100 compartments. This aggregated information corroborates the dominant role of the infiltration pattern on the spatial distribution of solute leaching. Interestingly, the maximum chloride concentration is quite independent of the amount of water captured by a sampling cell. It seems that there is very effective lateral spreading, at least within a few centimeters, since the lateral variation of the chloride concentration was relatively limited, even though flow rates varied massively. Based on these observations this suggests a convective-dispersive rather than stochastic-convective solute transport mechanism (Jury et al. 1991, pp. 222-225).

With the field measurement results we constructed solute flux concentration breakthrough curves $\mathrm{BTC}_{C}(x i, y i, t)$ for each compartment $(i, j)$ as well as the solute flux density breakthrough curves $\mathrm{BTC}_{F}(x i, y i, t)$ using Eq. 1. Figure $3 \mathrm{a}$, and b show five $\mathrm{BTC}_{C} \mathrm{~s}$ and $\mathrm{BTC}_{F} \mathrm{~s}$, respectively, out of the 100 curves generated by the sampling compartments. On the horizontal axis is cumulative drainage. The dominant role of the drainage pattern we observed in Fig. 2 is apparent here as well. The $\mathrm{BTC}_{F} \mathrm{~s}$, which are affected by variations in both solute concentration and volumes captured show much more variation than the $\mathrm{BTC}_{C} s$.

For each sampling compartment we determined the total amount of solute collected from it in the course of the experiment, and then we ranked the compartments and their BTCs in descending order of this collected solute amount. From these ranked 100 BTCs we constructed two leaching surfaces, one based on the $\mathrm{BTC}_{C} \mathrm{~s}$ and one based on the $\mathrm{BTC}_{F} \mathrm{~s}$ (Fig. 4). The leaching surface based on solute flux density shows that the amount of solute carried at any given time varies massively between cells, but the cells with low flow rates do not peak later than those with high flows, indicating efficient solute transfer between fast and slow domains. This solute transfer leads to much smaller variations in the concentrationbased leaching surface. The leaching surface based on solute flux density thus shows more variation than the leaching surface based on flux concentration because the variations in concentration and in amount of drainage are both represented in this graph. This figure shows the importance of the drainage in solute movement, as most of the mass is displaced within 
Day

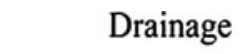

(mm)

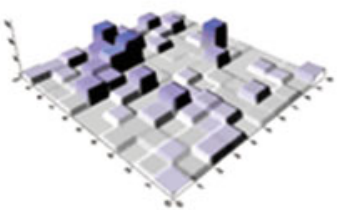

Day 20

Day 65
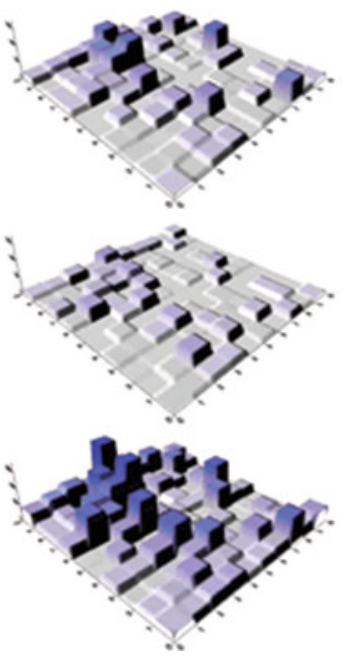

Concentration

chloride

(mg/l)
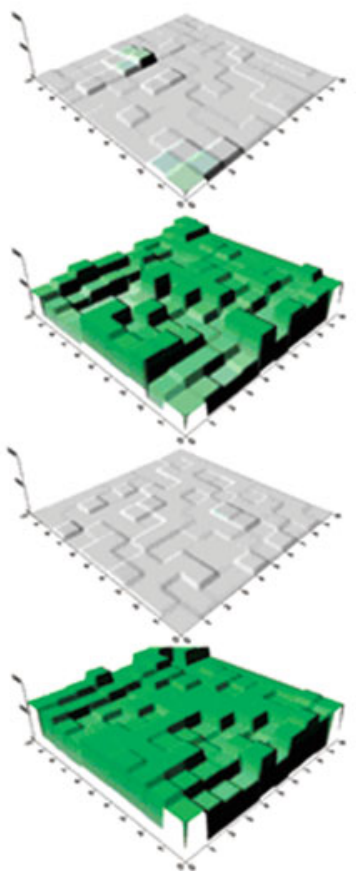

Mass

chloride

(mg)
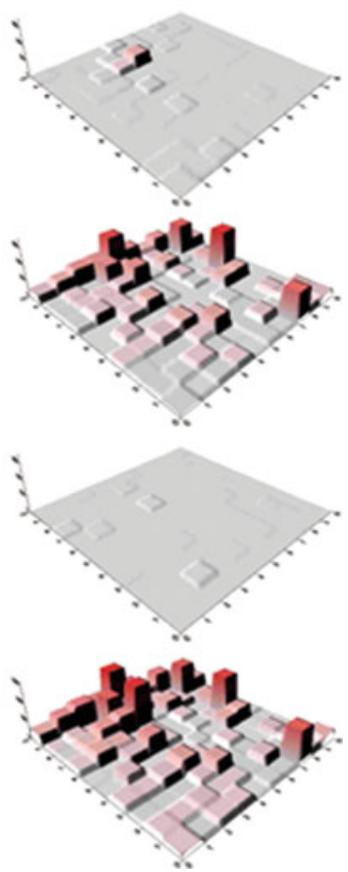

Fig. 2 Drainage, chloride concentration, and the amount of chloride for the sampled percolate in the sampler during sampling rounds on days 20,65, and 83 and the total drainage, maximum chloride concentration, and total mass measured over the whole sampling period
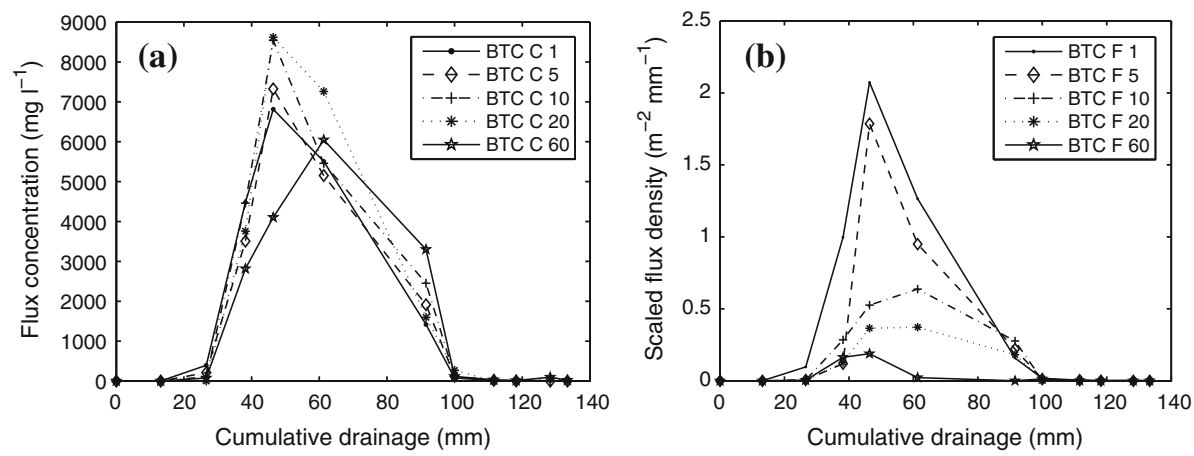

Fig. 3 Five BTCs based on flux concentrations (a) and from the same compartments the five BTCs based on solute flux density (b)

the cells with the highest fluxes, whereas the concentration is divided more or less equally over the whole sampling area. The area under each $\mathrm{BTC}_{C}$ based on flux concentration was determined (Fig. 5a), as well as the approximated SSDC (Fig. 5b). Figure 5b shows the Beta distribution fitted through the approximated SSDC. The fitted parameter values are in Table 2. 

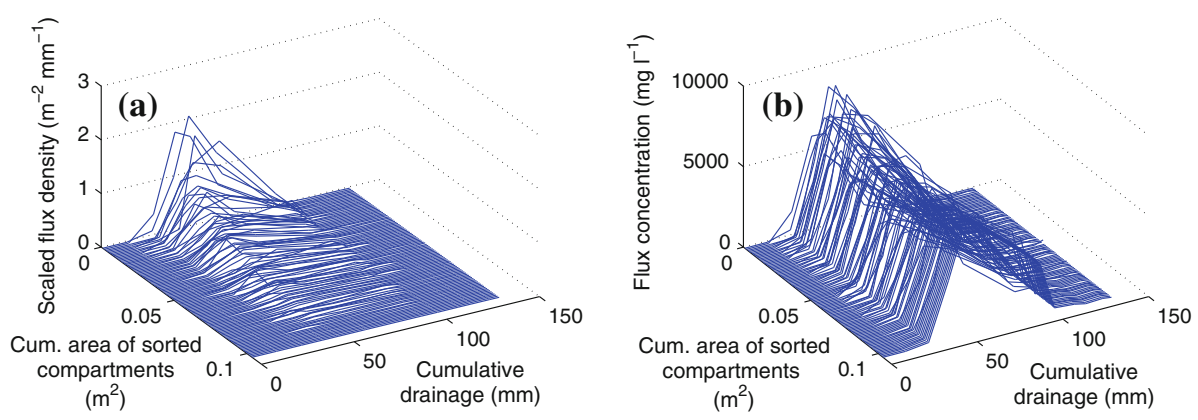

Fig. 4 Leaching surfaces for the tracer experiment based on scaled flux density (a) and based on flux concentration (b)
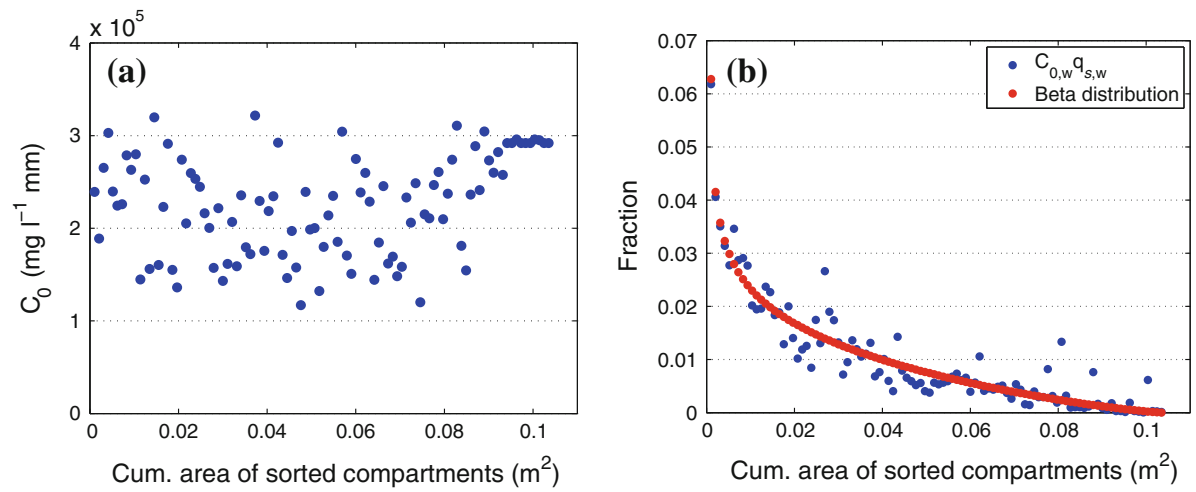

Fig. $5 \mathrm{C}_{0}$ is the area under each breakthrough curve based on flux concentration $\mathrm{BTC}_{C}(\mathbf{a})$, used to scale each BTC $_{C}$ to unity. To obtain the scaled flux density the scaled flux concentration $\mathrm{BTC}_{C}$ must be multiplied by $C_{0, \mathrm{w}} q_{s, \mathrm{w}}$. The fraction of this function is fitted by the Beta distribution (b)

Table 2 The mean, standard deviation and the coefficient of variation for the parameters velocity $v$ and dispersion coefficient $D$ fitted to Eq. 5 (Fig. 6), and the mean of $\alpha$ and $\zeta$ from the Beta distribution (Fig. 5) for a quantitative description of the leaching surface based on solute flux density

\begin{tabular}{lllll}
\hline & $\begin{array}{l}v \\
\left(\mathrm{~cm} \mathrm{~mm}^{-1}\right)\end{array}$ & $\begin{array}{l}D \\
\left(\mathrm{~cm}^{2} \mathrm{~mm}^{-1}\right)\end{array}$ & $\alpha$ & $\zeta$ \\
\hline Mean & 0.564 & 0.592 & 0.740 & 2.250 \\
STDEV & 0.043 & 0.123 & & \\
CV $(\%)$ & 7.69 & 20.73 & & \\
\hline
\end{tabular}

The BTC $_{C}$ s fitted by using Eq. 5 are given in Table 2 and Fig. 6. The error between the observed and fitted $\mathrm{BTC}_{C} \mathrm{~s}$ is acceptable given the low noise that is evident in Figs. 6, 7a,b and Table 3. The fitted velocities $v$ are fairly uniform, but seem to slightly decrease with $s$. The fits for $D$ are much more erratic without a clear trend. The velocities and dispersion coefficients are parameterized by one average velocity and one average dispersion coefficient as given in Table 2. This results in Fig. 7c. The error is slightly increased by $6 \%$ due to this operation. Applying the Beta distribution finally results in the fully parameterized leaching surface $S$ (Fig. 8c). 

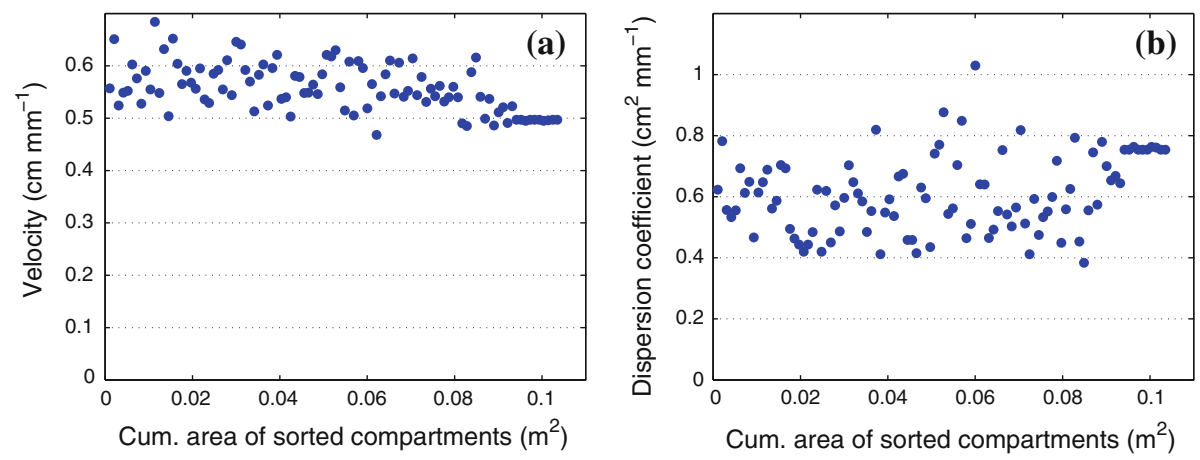

Fig. 6 The velocity $v(\mathbf{a})$ and dispersion coefficient $D(\mathbf{b})$ as fitted by Eq. 5 of the 100 sorted scaled BTC $C_{C} \mathrm{~s}$ based on flux concentration

Table 3 The normalized mean root mean square error (RMSE) (\%) between the observed and parameterized leaching surfaces based on flux concentration $\left(\mathrm{BTC}_{C}\right)$ and between the observed and parameterized leaching surfaces based on flux density $\left(\mathrm{BTC}_{F}\right)$

\begin{tabular}{lll}
\hline & Fit & Normalized mean RMSE (\%) \\
\hline BTC $_{C}$ & Direct & 0 \\
& $v$ and $D$ calculated & 6.7 \\
& $v$ and $D$ averaged & 12.7 \\
BTC $_{F}$ & Direct & 16.9 \\
& $v$ and $D$ calculated & 17.9 \\
& $v$ and $D$ averaged & 20.7 \\
\hline
\end{tabular}

For the leaching surfaces based on flux concentrations the errors have been calculated for the fitted $v$ and $D$, and for the averaged $v$ and $D$. For the scaled leaching surface based on flux density we calculated the error for the fit if the Beta distribution has been applied directly on the observed $\mathrm{BTC}_{F}$, applied on the fitted $v$ and $D$, and applied on the averaged $v$ and $D$

Figure 9 shows the leaching surface obtained when the observed BTCs were scaled according to the fitted Beta distribution, and thus solely reflects the effect of replacing the observed SSDC by its fitted counterpart. The errors of the fitted $\mathrm{BTC}_{C}$ s are $12.7 \%$. The error of the Beta distribution is $16.9 \%$.

Because trends in $v(s)$ and $D(s)$ are small or absent, we only applied the reference fit (with $v$ and $D$ equal to their mean for all $s$ ). This reduced the parameters describing the leaching surface to the four given in the top row of Table 2. The resulting leaching surface is shown in Fig. 8c. A comparison with the observed leaching surface (Fig. 8a) shows that the noise has been stripped to reveal the main features. The absence of a trend in $v$ and $D$ leads to a crest parallel to the $s$-axis and equally wide BTCs. The error for this leaching surface compared to the observed leaching surface is $20.7 \%$.

Of course, the fitted leaching surface is smooth. To reproduce some of the detailed features of the observed leaching surface we replaced the average values of $v$ and $D$ by randomly generated values from Gaussian distributions with mean and standard deviation given by the populations of fitted $v$ and $D$. We subdivided $s$ in equidistant intervals and assigned one set of the random values to each interval (qualitative approach). We used this qualitative approach for visualization. The obtained $v$ and $D$ values lead to scaled flux concentrations as presented in Fig. 7d. Visually the results for the scaled flux concentrations show a good resemblance 

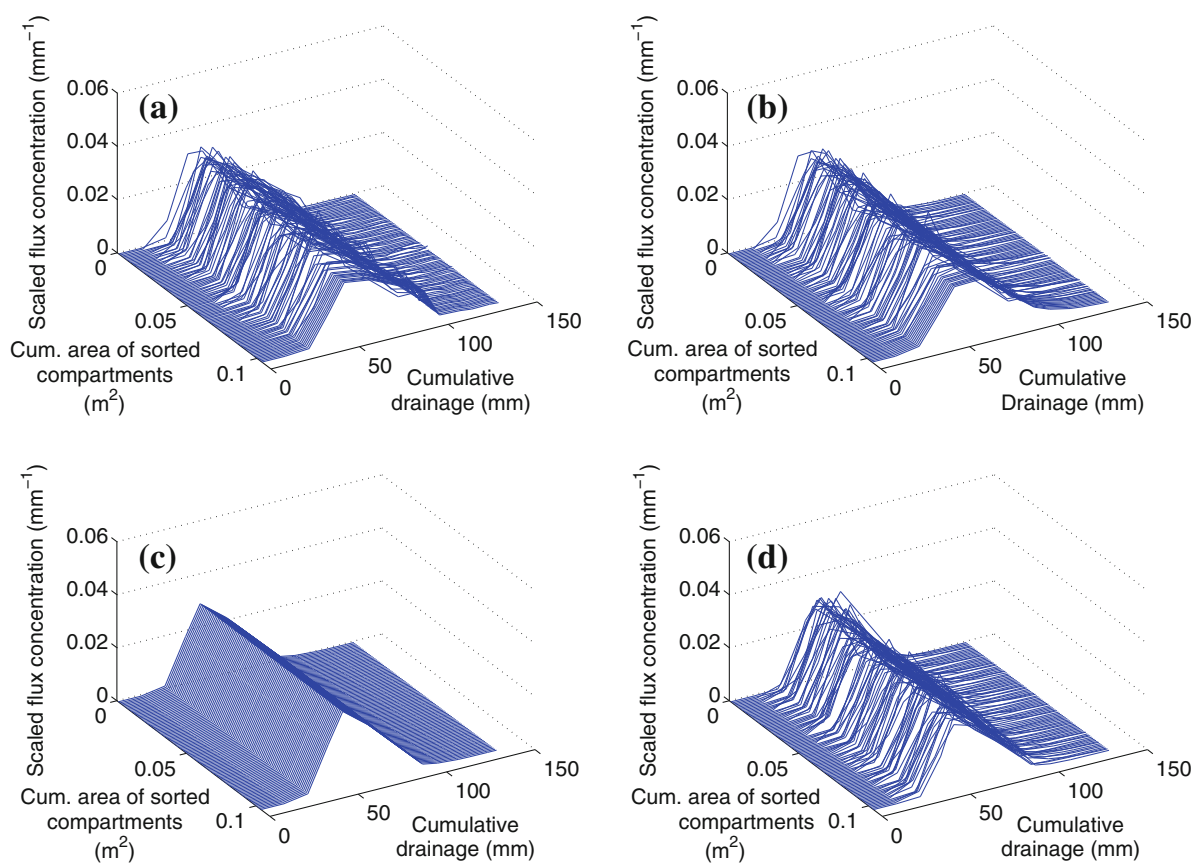

Fig. 7 The observed scaled leaching surface based on flux concentration (a), the scaled leaching surface based on flux concentration for the calculated $v$ and $D$ per BTC $C$, fitted to Eq. $5(\mathbf{b})$, the scaled leaching surface based on flux concentration with the average $v$ and average $D$ as given in Table 2 (c), and the scaled leaching surface based on flux concentration with a qualitative approach (d)

for observed scaled flux concentrations (Fig. 7a, d). Applying the Beta distribution give the qualitative leaching surface (Fig. 8d). In comparison with the smoothed leaching surface of Fig. 8c, the qualitative leaching surface gives a better visual result.

\section{Conclusions}

We developed a parameterization of the leaching surface that requires four to eight parameters. The parameterized form was fitted to an observed leaching surface. Main features, such as the concentration of leaching in a small region within the space-time domain, the marked spatial non-uniformity of the leaching and the relative similarity (apart from a scaling factor) of the BTCs of individual sampling cells, were represented rather well. This opens up the prospect of a quantitative comparison of leaching surfaces from different soils or obtained in different seasons. Possibly, leaching surface parameters can be used to characterize a local configuration of soil, climate, and land use in terms of its leaching behavior. This would be a more direct characterization than the currently used method of identifying soil hydraulic and transport properties to feed a numerical model that calculates leaching scenarios. The latter approach necessarily invokes all the assumptions that go into the separate solvers for the flow and transport equations, and the parameterizations upon which they rely. Furthermore, a parametric fit of a leaching surface allows the trend to be subtracted from the observations, thereby isolating the noise to allow an analysis of residuals. This analysis can prove a useful diagnostic of the leaching process: a few strong outliers with high leaching for instance point 

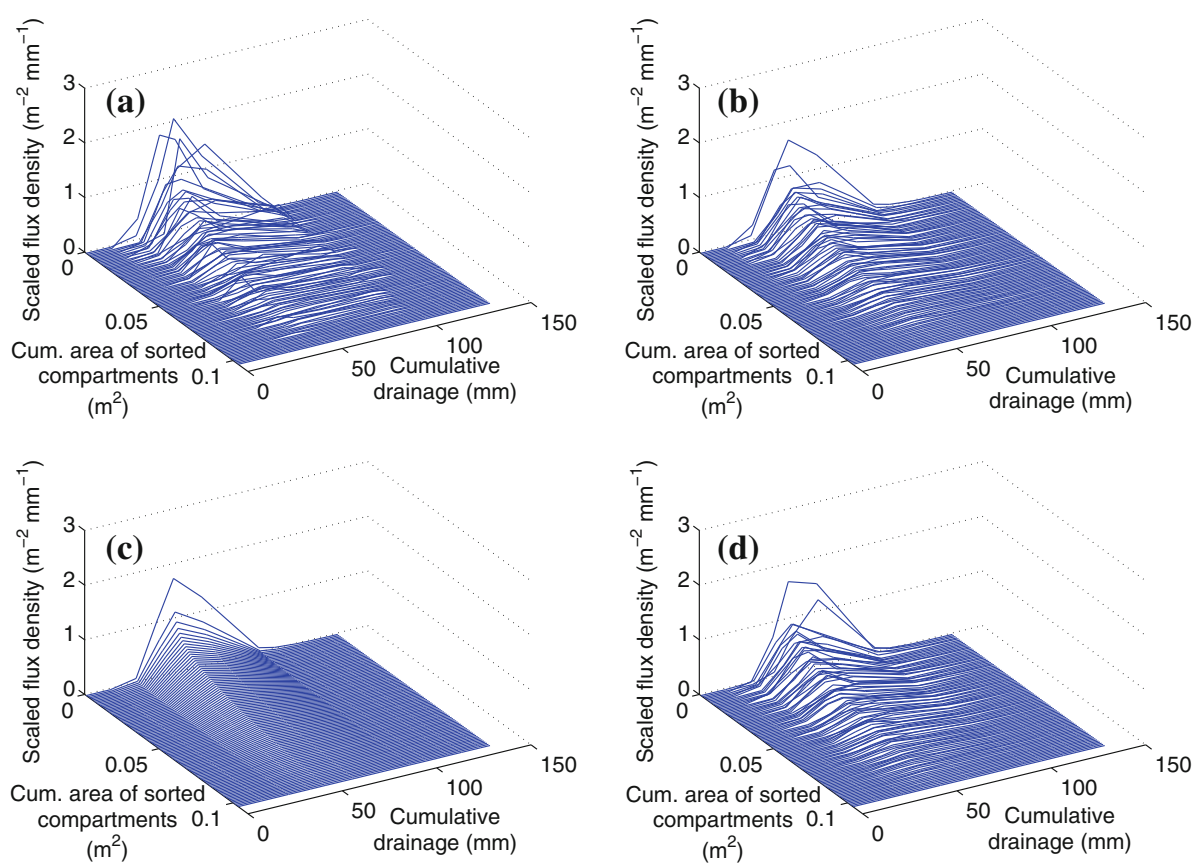

Fig. 8 The observed scaled leaching surface based on flux density (a) and parameterizations: scaled leaching surfaces based on flux density are constructed on the calculated $v$ and $D$ per $\mathrm{BTC}_{C}$ together with the fitted Beta distribution (b), the average $v$ and $D$ (Table 2) with the fitted Beta distribution (c), and a qualitative fit of $v$ and $D$ together with the fitted Beta distribution (d)

Fig. 9 The scaled leaching surface based on flux density constructed using the observed scaled BTC $_{C}$ s together with the fitted Beta distribution

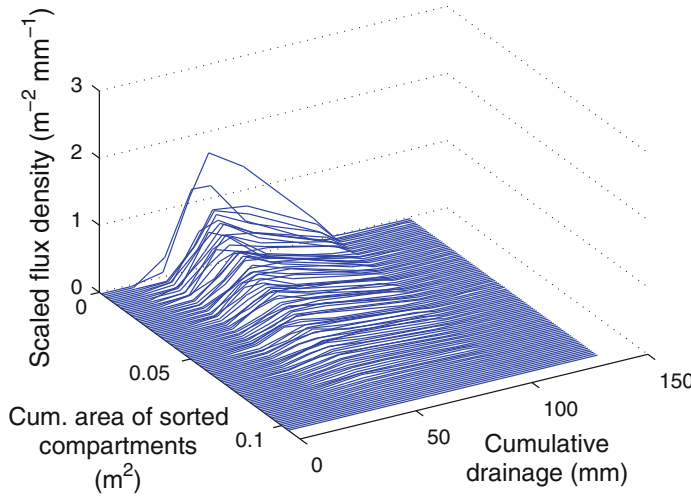

$\left(m^{2}\right)$

to the occurrence of narrow flow paths that funnel of sizable amounts of solute-amounts that can be actually quantified from the data. Another potential advantage is the fact that the leaching surface incorporates the effects of local heterogeneity on the leaching process and implicitly captures them in its parameter values. Thus, while soil cores obtained in a geostatistically homogeneous soil may produce vastly different BTCs, two MCSs buried in the same soil may generate leaching surfaces with comparable parameters. Thus, these parameters may characterize the leaching risks of relatively large areas, and spatial trends 
in them reflect significant differences, either caused by different land uses, or more gradual changes in soil properties, rainfall patterns, etc.

Acknowledgments This research is supported by the Research Council for Earth and Life Sciences (ALW) with financial aid from the Netherlands Organization for Scientific Research (NWO).

Open Access This article is distributed under the terms of the Creative Commons Attribution License which permits any use, distribution, and reproduction in any medium, provided the original author(s) and source are credited.

\section{References}

Beven, K.J., Henderson, D.E., Reeves, A.D.: Dispersion parameters for undisturbed partially saturated soil. J. Hydrol. 143, 19-43 (1993)

Bloem, E.: Variation in space and time of water flow and solute transport in heterogeneous soils and aquifers - a new multi-compartment percolation sampler and a new parameterization of the spatio-temporal solute distribution. PhD thesis. Wageningen University, Wageningen. ISBN 978-90-8504-922-7 (2008)

Bloem, E., Hogervorst, F.A.N., de Rooij, G.H.: A field experiment with variable-suction multi-compartment samplers to measure the spatio-temporal distribution of solute leaching in an agricultural soil. J. Contam. Hydrol. 105, 131-145 (2009). doi:10.1016/j.jconhyd.2008.11.010

Bloem, E., Hogervorst, F.A.N., de Rooij, G.H., Stagnitti, F.: Variable-suction multicompartment samplers to measure spatiotemporal unsaturated water and solute fluxes. Vadose Zone J. 9, 148-159 (2010). doi:10. 2136/vzj2008.0111

Brooks, M.C., Wise, W.R.: Quantifying uncertainty due to random errors for moment analyses of breakthrough curves. J. Hydrol. 303, 165-175 (2005)

Buchter, B., Hinz, C., Flury, M., Flühler, H.: Heterogeneous flow and solute transport in an unsaturated stony soil monolith. Soil Sci. Soc. Am. J. 59(1), 14-21 (1995)

de Rooij, G.H., Stagnitti, F.: Spatial variability of solute leaching: experimental validation of a quantitative parameterization. Soil Sci. Soc. Am. J. 64(2), 499-504 (2000)

de Rooij G.H., Stagnitti, F.: The solute leaching surface as a tool to assess the performance of multidimensional unsaturated solute transport models. In: Hassanizadeh, S.M., Schotting, R.J., Gray, W.G., Pinder, G.F. (eds.) Pre-conference proceedings of the international conference on computational methods in water resources, pp. 639-646. Delft, The Netherlands. Elsevier, Amsterdam (2002a)

de Rooij, G.H., Stagnitti, F.: Spatial and temporal distribution of solute leaching in heterogeneous soils: analysis and application to multisampler lysimeter data. J. Contam. Hydrol. 54, 329-346 (2002b)

de Rooij, G.H., Stagnitti, F.: Applications of the beta distribution in soil science. In: Gupta, A.K., Nadarajah, S. (eds.) Handbook of Beta distribution and its applications, pp. 535-550. Marcel Dekker Inc., New York (2004)

Fahim, M.A., Wakao, N.: Parameter estimation from tracer response measurements. Chem. Eng. J. 25, $1-8(1982)$

Flury, M., Wai, N.N.: Dyes as tracers for vadose zone hydrology. Rev. Geophys. 41(1), 2.1-2.37, 1002 (2003). doi:10.1029/2001RG000109

Flury, M., Flühler, H., Jury, W.A., Leuenberger, J.: Susceptibility of soils to preferential flow of water: a field study. Water Resour. Res. 30(7), 1945-1954 (1994)

Gelhar, L.W., Welty, C., Rehfeldt, K.R.: A critical review of data on field-scale dispersion in aquifers. Water Resour. Res. 28(7), 1955-1974 (1992)

Gupta, A.K., Nadarajah, S.: Mathematical properties of the beta distribution. In: Gupta, A.K., Nadarajah, S. (eds.) Handbook of Beta distribution and its applications, pp. 33-53. Marcel Dekker Inc., New York (2004)

Haas, C.N., Joffe, J., Heath, M.S., Jacangelo, J.: Continuous flow residence time distribution function characterization. J. Environ. Eng. 123(2), 1121-1123 (1997)

Jury, W.A., Roth, K.: Transfer functions and solute movement through soil, 226 pp. Birkhauser Verlag, Basel (1990)

Jury, W.A., Sposito, G.: Field calibration and validation of solute transport models for the unsaturated zone. Soil Sci. Soc. Am. J. 49, 1331-1341 (1985)

Jury, W.A., Gardner, W.R., Gardner, W.H.: Soil Physics. Wiley, New York (1991)

Kasteel, R., Vogel, H.J., Roth, K.: Effect of non-linear adsorption on the transport behaviour of brilliant blue in a field soil. Eur. J. Soil. Sci. 53, 231-240 (2002) 
Kool, J.B., Parker, J.C., van Genuchten, M.Th.: Parameter estimation for unsaturated flow and transport models - a review. J. Hydrol. 91, 255-293 (1987)

Kreft, A., Zuber, A.: On the physical meaning of the dispersion equation and its solutions for different initial and boundary conditions. Chem. Eng. Sci. 33, 1471-1480 (1978)

Mishra, S., Parker, J.C.: Parameter estimation for coupled unsaturated flow and transport. Water Resour. Res. 25(3), 385-396 (1989)

Nadarajah, S., Gupta, A.K.: Beta funtion and the incomplete beta function. In: Gupta, A.K., Nadarajah, S. (eds.) Handbook of Beta distribution and its applications, pp. 1-31. Marcel Dekker Inc., New York (2004)

Parker, J.C., van Genuchten, M.Th.: Flux-averaged and volume-averaged concentrations in continuum approaches to solute transport. Water Resour. Res. 20(7), 866-872 (1984)

Persson, M.: Accurate dye tracer concentration estimations using image analysis. Soil Sci. Soc. Am. J. 69(4), 967-975 (2005)

Poletika, N.N., Jury, W.A.: Effects of soil surface management on water flow distribution and solute dispersion. Soil Sci. Soc. Am. J. 58(4), 999-1006 (1994)

Quisenberry, V.L., Phillips, R.E., Zeleznik, J.M.: Spatial distribution of water and chloride macropore flow in a well-structured soil. Soil Sci. Soc. Am. J. 58(5), 1294-1300 (1994)

Stagnitti, F., Sherwood, J., Allinson, G., Evans, L., Allinson, M., Li, L., Phillips, I.: An investigation of localised soil heterogeneities on solute transport using a multisegment percolation system. New Zealand J. Agricul. Res. 41, 603-612 (1998)

Stagnitti, F., Li, L., Allinson, G., Phillips, I., Lockington, D., Zeiliguer, A., Allinson, M., Lloyd-Smith, J., Xie, M.: A mathematical model for estimating the extent of solute- and water-flux heterogeneity in multiple sample percolation experiments. J. Hydrol. 215, 59-69 (1999)

Strock, J.S., Cassel, D.K., Gumpertz, M.L.: Spatial variability of water and bromide transport through variably saturated soil blocks. Soil Sci. Soc. Am. J. 65(6), 1607-1617 (2001)

Toride, N., Leij, F.J., van Genuchten, M.Th.: The CXTFIT code for estimating transport parameters from laboratory or field tracer experiments. Version 2.1. Tech. Rep. Research Report No. 137, U. S. Salinity Laboratory, Agricultual Research Service, U. S. Department of Agriculture, Riverside (1999)

Vanderborght, J., Vereecken, H.: Analyses of locally measured bromide breakthrough curves from a natural gradient tracer experiment at Krauthausen. J. Contam. Hydrol. 48, 23-43 (2001)

van Ommen, H.C., van Genuchten, M.T., van der Molen, W.H., Dijksma, R., Hulshof, J.: Experimental and theoretical analysis of solute transport from a diffuse source of pollution. J. Hydrol. 105, 225-251 (1989)

Wildenschild, D., Jensen, K.H., Villholth, K., Illangasekare, T.H.: A laboratory analysis of the effect of macropores on solute transport. Gr. Water 32(3), 381-389 (1994)

Yamaguchi, T., Moldrup, P., Yokosi, S.: Using breakthrough curves for parameter estimation in the convection-dispersion model of solute transport. Soil Sci. Soc. Am. J. 53, 1635-1641 (1989) 